\title{
Dynamic Compressive Spectrum Sensing for Cognitive Radio Networks
}

\author{
Wotao Yin ${ }^{1}$, Zaiwen Wen ${ }^{3}$, Shuyi $\mathrm{Li}^{1}$, Jia (Jasmine) Meng ${ }^{2}$, and Zhu Han ${ }^{2}$ \\ ${ }^{1}$ Department of Computational and Applied Mathematics, Rice University \\ ${ }^{2}$ Department of Electrical and Computer Engineering, University of Houston \\ ${ }^{3}$ Department of Mathematics and Institute of Natural Sciences, Shanghai Jiaotong University
}

\begin{abstract}
In the recently proposed collaborative compressive sensing, the cognitive radios (CRs) sense the occupied spectrum channels by measuring linear combinations of channel powers, instead of sweeping a set of channels sequentially. The measurements are reported to the fusion center, where the occupied channels are recovered by compressive sensing algorithms. In this paper, we study a method of dynamic compressive sensing, which continuously measures channel powers and recovers the occupied channels in a dynamic environment. While standard compressive sensing algorithms must recover multiple occupied channels, a dynamic algorithm only needs to recover the recent change, which is either a newly occupied channel or a released one. On the other hand, the dynamic algorithm must recover the change just in time. Therefore, we propose a least-squared based algorithm, which is equivalent to $\ell_{0}$ minimization. We demonstrate its fast speed and robustness to noise. Simulation results demonstrate effectiveness of the proposed scheme.
\end{abstract}

\section{INTRODUCTION}

Today, most spectrum is used ineffectively, and their utilization varies by time and location. To increase spectrum utilization, cognitive radios (CRs) [1] are used to detect and share the unused spectrum. However, each CR has a limited reach, and channel scan is time consuming. Inspired by compressive sensing [3], a collaborative spectrum sensing method was proposed in [4] to resolve these problems. Instead of sweeping channels sequentially, each CR is equipped with a few frequency selective filters, through which a number of linear combinations of the powers of all channels are recorded and then sent to the fusion center (FC). The FC discovers the occupied channels from the $\mathrm{CR}$ reports by running a recovery algorithm. This algorithm exploits a "joint sparsity" property of the channel-power vectors from different CRs: only a few channels that are occupied have high powers, so the vector at each CR is sparse; meanwhile, every occupied channel is recorded by multiple CRs, so the CRs see the high powers on a common set of channels. This set of jointly sparse power vectors are recovered to reveal the occupied channels. The work [4] allows the communications between the CRs and FC to be lossy. Missing or erroneous reports can be accurately recovered by matrix completion (see [6], [9]) since the matrix formed by all reports has a low rank. Hence, the detection is robust to errors. In comparison with traditional spectrum sensing, this method reduces the workload of sensing and communication.

However, the work [4] only discusses spectrum sensing in a static environment. In the real world, PRs may start or stop using their channels at any time. In order to detect changes in the spectrum usage in a dynamic environment, this paper studies a dynamic compressive sensing system, as well as its detection method. In the proposed system, the channel occupancy status and channel gains evolve, and CR reports are sent continuously to the FC. We show that given existing channel occupancy information, updating this information can be quick and reliable.

The rest of this paper is organized as follows: Related work is studied in Section II. In Section III, the system model is given. Section IV studies the dynamic sensing scheme and propose an algorithm. Simulation results are given in Section V. Finally, Section VI concludes this paper.

\section{RELATED WORK}

\section{A. Existing Work on Collaborative Spectrum Sensing}

It has been shown that, through collaborative spectrum sensing (CSS) among CRs, the effects of hidden terminal problem can be reduced and the probability of detecting the primary radio (PR) can be improved [11]-[17]. In [11], the CRs collaborate by sharing their sensing decisions through an FC, which combines the CRs' sensing bits using the ORrule for data fusion. A similar approach is used in [12] using different decision-combining methods. In [13], an evolutionary game model for CSS is proposed in order to inspect the strategies of the CRs and their contribution to the sensing process. In [14], spatial diversity techniques are proposed for improving the performance of CSS by combatting the error probability due to fading on the reporting channel between the CRs and the FC. Other performance aspects of CSS are studied in [15]-[17].

\section{B. Related Work based on Compressive Sensing}

There are several recent applications of compressive sensing in wireless communications. In [18], a two-step compressed spectrum sensing scheme for efficient wideband sensing is proposed. In [19], a Bayesian compressive sensing framework reduces the sampling requirement and computational complexity by bypassing signal reconstruction. In [20], to collect spatial diversity against wireless fading, multiple CRs collaborate and establish consensus among local spectral estimates through running a decentralized consensus optimization algorithm. In [21], compressive sampling is performed at local CRs to scan wide spectra, and measurements from multiple CR detectors 
are fused to collect spatial diversity gain, which improves the detection quality, in particular, under fading channels.

\section{Related Work on Dynamic Compressive Sensing}

Dynamic compressive sensing work [22]-[24] develops efficient algorithms for updating the solutions of $l_{1}$ minimization for streaming measurements, where the underlying signal changes and new measurements of a fixed signal are sequentially added to the system. The proposed algorithms are based on homotopy continuation, which breaks down the solution update into a small sequence of linear steps. The idea of [25] is to estimate the initial signal support by compressive sensing, run a reduced order Kalman filter over the estimated support, and apply compressive sensing to the Kalman innovations or filtering error upon any change occurs to the support.

\section{Compressive SPectrum Sensing Model}

We consider a cognitive radio network with $M$ CRs monitoring $N$ channels, within which there are $S$ occupied channels. For simplicity, we assume that each occupied channel corresponds to one PR. Each $\mathrm{CR}$ is equipped with $P$ frequency selective filters, which continuously record $P$ different linear combinations of the powers of all the $N$ channels. Their reports are sent to the FC continuously at a certain rate. Here, $P$ and $M$ are much smaller than $N$. Hence the system requires a smaller amount of sensing and transmission compared to the traditional approach. The formation of the reports can be described with three matrices $A \in \mathbb{R}^{P \times N}, R \in \mathbb{R}^{N \times N}$ and $G \in \mathbb{R}^{M \times N}$. The entries of $A$ are frequency filter coefficients used by the CRs to generate $P$ reports. Each CRs can have its own unique $A$ but we assume a uniform $A$ just for notational simplicity. $R$ is a diagonal matrix with 1 or 0 on its diagonal, where $R_{n n}=1$ means that channel $n$ is occupied and 0 means unoccupied. $G$ represents the channel gains between the CRs which are given as

$$
G_{m, n}=\left(d_{m, n}\right)^{-\alpha / 2}\left|h_{m, n}\right|,
$$

where $d_{m, n}$ is the distance between the $m^{t h} \mathrm{CR}$ and the primary user using the $n^{\text {th }}$ channel, $\alpha$ is the propagation loss factor, and $h_{m, n}$ is the channel fading. The CR reports $B$ are given as

$$
B=A\left(R G^{\top}\right)=A X \in \mathbb{R}^{P \times M}, \text { where } X=R G^{\top} .
$$

The goal of (static) spectrum sensing is to recover $X$ from a subset of entries in $B$. In $X$, each column $m$ (i.e. $X_{:, m}$ ) corresponds to the channel power received by $\mathrm{CR} m$, and each row $X_{n, \text { : }}$ corresponds to the powers of channel $n$. All but $S$ rows of the matrix $X$ are zero. These nonzero rows correspond to the occupied channels. Since there are only a small number of used channels, $X$ is sparse in terms of nonzero rows. In each nonzero row $X_{n,:}$, there is typically more than one nonzero; if $X_{n, m} \neq 0$, other entries in the same row are likely nonzero. This joint sparsity property allows $X$ to be recovered from a small number of entries in $B$ by algorithms such as [7], [8].
The algorithm in [4] takes into consideration the large dynamic range of channel powers so it returns more stable recovery.

In a dynamic environment, $X$ changes continuously. This paper considers discrete-times changes, which arise when a certain PR starts or stops using a channel, or the power of a channel changes significantly. Each change corresponds to a change to one certain row of $X$. In the future, we will study cases in which multiple changes can happen simultaneously. The dynamic spectrum sensing problem is divided into a sequence of time periods that are separated by the changes. Let $B_{\text {prev }}$ and $X_{\text {prev }}$ denote $B$ and $X$ in the previous period. In the current period, we shall recover $X$ given $X_{\text {prev }}$ and $B_{\text {prev }}$, as well as the entries of $B$ that are received since the start of this period. From this $X$, the current occupied channels and their gains can be computed. Since each period requires solutions from its previous period, the success of dynamic recovery is based on the success in every period, which is in turn based on the received entries in $B$ in every period. First, let us set up the model for the current period.

Let $n^{*}$ be the index of the changed row of $X$ since the previous period and let $A=\left[a_{1}, \cdots, a_{N}\right]$. Then, $\Delta B=B-$ $B_{\text {prev }}=A X-A X_{\text {prev }}=a_{n^{*}}(\Delta X)_{n^{*},:}$, where $(\Delta X)_{n^{*},:}$ is the $n^{* t h}$ row of $\Delta X$. Let $\Omega$ denote the set of the received entries of $B$ (equivalently, those of $\Delta B$ ). The problem is to recover $n^{*}$ and $(\Delta X)_{n^{*},:}$ from the given $A$ and the received entries $(\Delta B)_{p, m},(p, m) \in \Omega$ following the system model

$$
\Delta B=a_{n^{*}}(\Delta X)_{n^{*},:}
$$

\section{RECOVERY METHOD}

The underlying problem is joint-sparsity recovery of only one nonzero row. There is no need to use techniques such as $\ell_{1}$ minimization or greedy pursuit algorithms. In stead, the recovery can be as simple as solving the least-squares problem

$$
\min _{n \in\{1, \ldots, N\}} \min _{y_{n}}\left\|\left(a_{n} y_{n}\right)_{\Omega}-(\Delta B)_{\Omega}\right\|_{2},
$$

where $y_{n}$ is a row vector with $M$ entries (thus $\left(a_{n} y_{n}\right) \in$ $\mathbb{R}^{P \times M}$ ) and the subscripted notion $X_{\Omega}$ stands for the vector formed by the entries $X_{p, m},(p, m) \in \Omega$. Noting that problem (3) seeks a row $n$ of $\Delta X$ that best matches the observation $(\Delta B)_{\Omega}$, we can see that $n=n^{*}$ and $y_{n^{*}}=(\Delta X)_{n^{*},:}$ attain the minimum zero objective in (4).

\section{A. Parallel Implementation}

For convenience of linear algebra, define $I_{m}:=\{p$ : $(p, m) \in \Omega\}$, which is the set of $B$ entries that are received from the $m^{t h} \mathrm{CR}$. Then, the objective function of (4) can be written as

$$
\left\|\left(a_{n} y_{n}\right)_{\Omega}-(\Delta B)_{\Omega}\right\|_{2}^{2}=\sum_{m=1}^{M}\left\|\left(y_{n}\right)_{m} a_{I_{m}, n}-(\Delta B)_{I_{m}, m}\right\|_{2}^{2},
$$

where for each $m$ and $n$ given, $a_{I_{m}, n}$ is the column vector formed by $\left\{a_{p, n}:(p, n) \in \Omega\right\}$ and $(\Delta B)_{I_{m}, m}$ is the column 
vector formed by $\left\{(\Delta B)_{p m}:(p, m) \in \Omega\right\}$. Given $k$, the minimizer of (5) is

$$
\left(y_{n}^{*}\right)_{m}=\frac{\left(a_{I_{m}, n}\right)^{\top}(\Delta B)_{I_{m}, m}}{\left(a_{I_{n}, m}\right)^{\top} a_{I_{n}, m}}, \quad m=1, \ldots, M .
$$

For fast computation, we propose a vectorized algorithm, which returns $Y^{*} \in \mathbb{R}^{N \times M}$ consisting of all row vectors $y_{n}^{*}$, $n=1, \ldots, N$. We introduce

$$
\begin{array}{cl}
\overline{\Delta B} \in \mathbb{R}^{P \times M}, & (\overline{\Delta B})_{p, m}= \begin{cases}(\Delta B)_{p, m}, & (p, m) \in \Omega \\
0, & \text { otherwise; }\end{cases} \\
P \in \mathbb{R}^{P \times M}, & P= \begin{cases}1, & (p, m) \in \Omega, \\
0, & \text { otherwise; }\end{cases} \\
\hat{A} \in \mathbb{R}^{P \times N}, & \hat{A}_{p, n}=A_{p, n}^{2} .
\end{array}
$$

Then, the numerator $\left.\mathbf{a}_{I_{m}, n}\right)^{\top}(\Delta B)_{I_{m}, m}=\left(A^{\top} \overline{\Delta B}\right)_{n, m}$ and the denominator $\left(\mathbf{a}_{I_{m}, n}\right)^{\top} \mathbf{a}_{I_{m}, n}=\left(\hat{A}^{\top} P\right)_{n, m}$. Therefore, we have

$$
Y^{*}=\frac{A^{\top}(\overline{\Delta B})}{\hat{A}^{\top} P} \quad \text { (component-wise division). }
$$

Formula (10) can be computed by an advance matrix-matrix multiplication algorithm [10] with sub-cubic complexity.

Note that for (10) to be well-defined, $\Omega$ must has one entry for each CR, i.e., $\{p:(p, m) \in \Omega\} \neq \emptyset$ for $m=1, \ldots, M$. Under this condition, all entries of $\hat{A}^{\top} P$ are nonzero for all practical choices of compressive sensing matrices $A$.

To solve (4) over $n$, one shall compare

$$
\left\|\left(a_{n} y_{n}^{*}\right)_{\Omega}-(\Delta B)_{\Omega}\right\|_{2}, n=1, \ldots, N
$$

where $y_{n}^{*}$ is the $n^{\text {th }}$ row of $Y^{*}$. If there is a unique $n$ that gives 0 or a tiny value, then this $n$ is $n^{*}$, the row that has changes since the previous period. Therefore, let

$$
X_{n,:} \leftarrow\left(X_{\text {prev }}\right)_{n,:}+\left\{\begin{array}{ll}
y_{n}^{*}, & n=n^{*} ; \\
0, & \text { otherwise, }
\end{array} \quad n=1, \ldots, N .\right.
$$

The complexity of (11) is $O(|\Omega| N)$ and that of (12) is $O(N)$, assuming an in-place update from $X_{\text {prev }}$ to $X$.

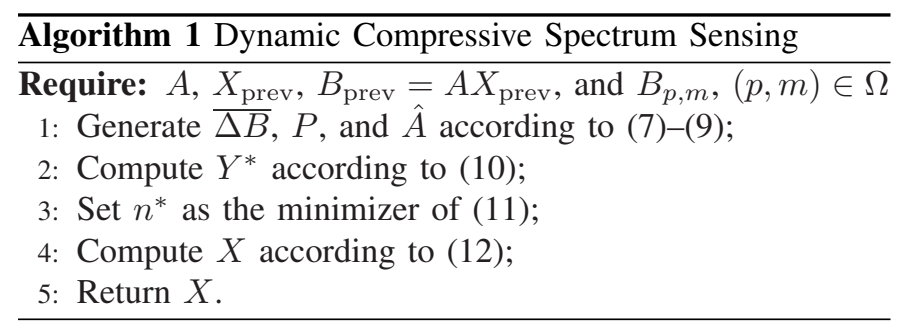

\section{B. Required Measurements}

This subsection studies the required measurements for the correct recovery of $X$. Let $\Omega_{m}=\Omega \cap\{(p, m): p=1, \ldots, P\}$.

Theorem 1: Assume that both $X_{\text {prev }}$ and $B_{p, m},(p, m) \in \Omega$, are exact, and the entries of $A$ are in general positions ${ }^{1}$. If

\footnotetext{
${ }^{1}$ Any $p$ distinct subsets of $\left\{A_{i j}\right\}$ of length $q \geq p$ are linearly independent.
}

$\Omega_{m} \neq \emptyset, m=1, \ldots, M$, and there exists at least one $m$ such that $\left|\Omega_{m}\right| \geq 2$, then $X$ given by Algorithm (1) is exact.

Proof: First, $n=n^{*}$ and $y_{n^{*}}=(\Delta X)_{n^{*}, \text { : }}$ attain the minimum zero objective in (4). Second, without loss of generality, assume $\left|\Omega_{1}\right| \geq 2$. Since the entries of $A$ are in general positions, the term $\left\|\left(y_{n}\right)_{1} a_{I_{1}, n}-(\Delta B)_{I_{1}, 1}\right\|_{2}^{2}$ in (5) can reach zero, the minimal possible value, only for $n=n^{*}$. Combining the two points, we conclude that Algorithm 1 returns $n=n^{*}$. Finally, since every $\Omega_{m}$ is nonempty, every entry of $\left(y_{n^{*}}\right)_{m}$ that minimizes the term $\left\|\left(y_{n^{*}}\right)_{m} a_{I_{m}, n^{*}}-(\Delta B)_{I_{m}, m}\right\|_{2}^{2}$ in (5) is unique and equal to $(\Delta X)_{n^{*}, m}$.

In our future work or a future version of this paper, we will consider a realistic noise scenario and provide the number of measurements for the stable recovery of $\Delta X$.

\section{Dynamic vs Static Compressive Sensing}

In a dynamic environment, dynamic compressive sensing is not only simpler but also requires fewer measurements than standard (static) compressive sensing. Our model of dynamic compressive sensing recovers $\Delta X$, which has only one nonzero row. This is a special case of static compressive sensing where one recovers multiple nonzero rows. With one nonzero row, one can afford an exhaustive search for that row, which requires $O(M)$ measurements and is computationally as cheap as solving $N$ simple least-squares problems (which can be solved in parallel, using fast matrix computation, or utilizing group testing). This is equivalent to performing the $\ell_{0}$-minimization over the rows (the $\ell_{0}$ "norm" measures the number of nonzero entries). It is well known that for seeking solutions with a general number of nonzero rows, $\ell_{0}$-minimization is NP-hard. The $\ell_{1}$-minimization, as the best convex approximation of $\ell_{0}$, requires $O(L \log (N / L))$ measurements and runs much slower than $N$. Therefore, dynamic compressive sensing is a much better choice in terms of measurements and computing time required. On the other hand, the success of dynamic compressive sensing requires an accurate previous solution. In case dynamic compressive sensing fails due to either lack of measurements or inaccurate previous solution, one must gather more measurements and fall back on static compressive sensing.

\section{Simulations}

In this section, we perform numerical simulations to illustrate the performance of the proposed dynamic spectrum sensing algorithm. We study the ability of instant capture of the change in channel occupancy status. As long as no two changes happen simultaneously, our algorithm should be able to detect them one by one. We report the rate of detection success defined by the number of correct detections over the total number 500 of randomly generated instances in which only one channel status changed.

\section{A. Simulation Settings}

Our experiments were based on a cognitive radio network with 100 channels, and $20 \mathrm{CR}$ nodes performing collaborative spectrum sensing. The simulation used two types of channel 
model: type 1, Rayleigh fading channel; and type 2 Lognormal shadowing channel. The number of occupied channels varied from 2 to 20. Each time, we either added or deleted an occupied channel to test if our algorithm could detect the change correctly. The results for significant power change in an occupied channel was similar, so we do not plot them. The SNRs of $\Delta B$ were chosen between $10 \mathrm{~dB}$ and $30 \mathrm{~dB}$.

\section{B. SNRs of $\Delta B$}

Our simulations were done with different SNRs of $\Delta B$. In this subsection, we discuss several factors that can affect the SNR of $\Delta B$. The SNR of $\Delta B$ depends on the those of $B_{\text {prev }}$ and $B$, as well as the powers of the changed channel.

The SNR of $B_{\text {prev }}$ further depends on the level of thermal noise, the accuracy of the recovered $X_{\text {prev }}$ (as not all entries of $B_{\text {prev }}$ are directly received), as well as the length of the previous time period, during which there is no significant change in the channel powers. The SNR of $B_{\text {prev }}$ usually improves over time as more measurements are received and noise get averaged over time. Hence, the longer the length of a stationary period, the higher the SNR. Once a change occurs, the new time period starts, and the FC receives entries of $B$. The SNR of $B$ also depends on the thermal noise level and the number of measurements received. However, the missing entries of $B$ are not used.

For fixed SNRs of $B_{\text {prev }}$ and $B$, the SNR of $\Delta B$ will be higher if the power change (i.e., the signal of $\Delta B$ ) is stronger, which translates to the unique nonzero row of $\Delta X$ containing larger values. Physically, if a nearby or high-power PR starts or stops, the SNR of $\Delta B$ will be large. On the contrary, if a distant or low-power PR starts or stops, the SNR of $\Delta B$ will be small.

Since the SNR of $\Delta B$ depends on several factors, it is tedious to run simulations with all the factors varying. Instead, we directly varied the SNRs of $\Delta B$.

\section{Dynamic Compressive Sensing Algorithm Performance}

In Figure 1, we show the rate of detection success versus the sampling rate, which is defined as the number of received entries of $\Delta B$ over its total number of entries. We varied the sampling rate from $5 \%$ to $40 \%$. Note that $\Delta B$ is already much compressed compared to $X$, and the reference of our sampling rate is to the size of $\Delta B$, not that of $X$.

Figures 1 (a) and (b) correspond to the performance for SNR $=20 \mathrm{~dB}$ and $\mathrm{SNR}=30 \mathrm{~dB}$, respectively. When $\mathrm{SNR}=20 \mathrm{~dB}$ (shown in 1 (a)), we only need less than $25 \%$ of the samples at the fusion center to guarantee a successful detection.Neither channel model nor add/drop type affects the overall detection performance. As SNR increased to 30dB (shown in 1 (b)), the sampling rate can be further reduced to $25 \%$ for reliable detection.

Figure 2 shows the successful detection rate of both adding and deleting one occupied channel as the number of occupied channel changes. In these simulations, we fix the SNR at $30 \mathrm{~dB}$. There is no obvious change in the detection rate as the number of occupied channel increases from 2 to 20 in both cases.

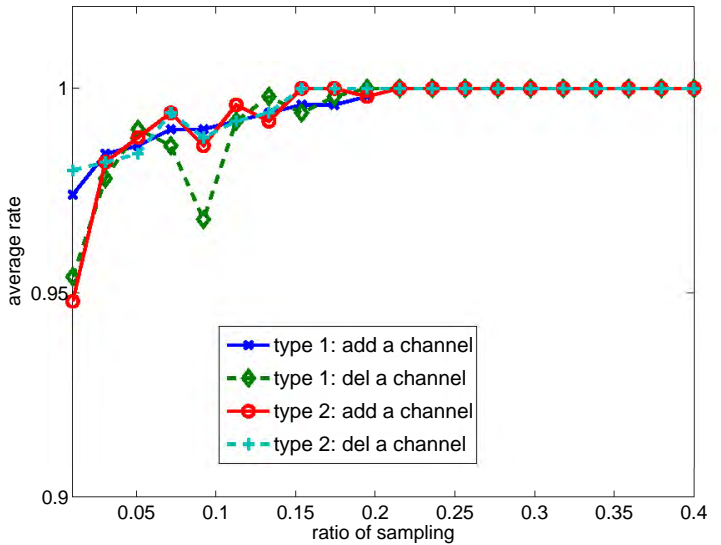

(a) $\mathrm{SNR}=20$

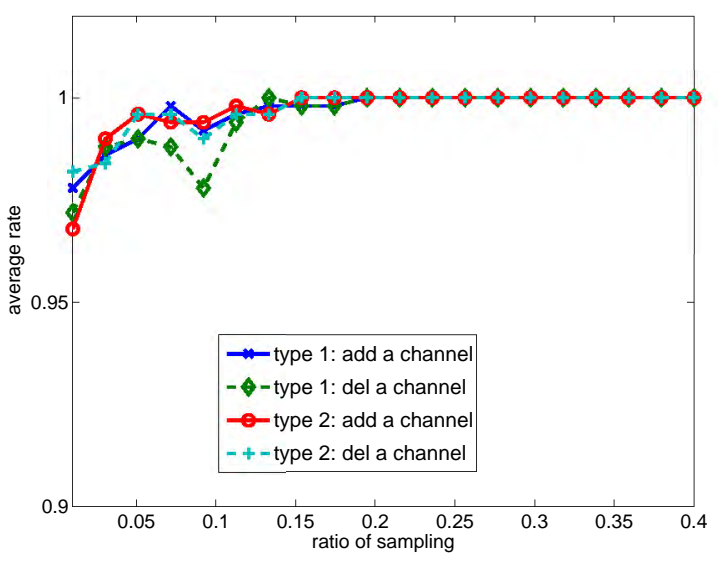

(b) $\mathrm{SNR}=30$

Fig. 1. Average rate (500 runs each point) of correct recovery for $p=30$ reports, $n=100$ total channels, $m=20 \mathrm{CRs}, s=4$ occupied channels before change.

Again, the channel models do not affect the detection rate, no matter there is increase or decrease of the number of occupied channels.

Figure 2 (a) shows a tough situation, in which, only $10 \%$ of the reports have been captured by the FC. We have bumpy curves here. When the situation becomes better, i.e., the FC received $20 \%$ of the reports from the CRs, the over all detection performance becomes more stable (shows in Figure 2 (b)).

Figure 3 depicts the detection rate at different SNR when we fix the number of occupied channels. Comparing Figure 3 (a) and (b), we can tell that the increase in the number of occupied channel makes it harder to detect the changes. When the SNR is low and number of occupied channel is high, the detection rate will be relatively low.

\section{Vi. Conclusions and Future Directions}

In order to reduce the amount of sensing and transmission workload of cognitive radio (CR) nodes, we have applied compressive sensing for collaborative spectrum sensing in cognitive radio networks. In this paper, we move from a static environment to a dynamic one where channel powers 


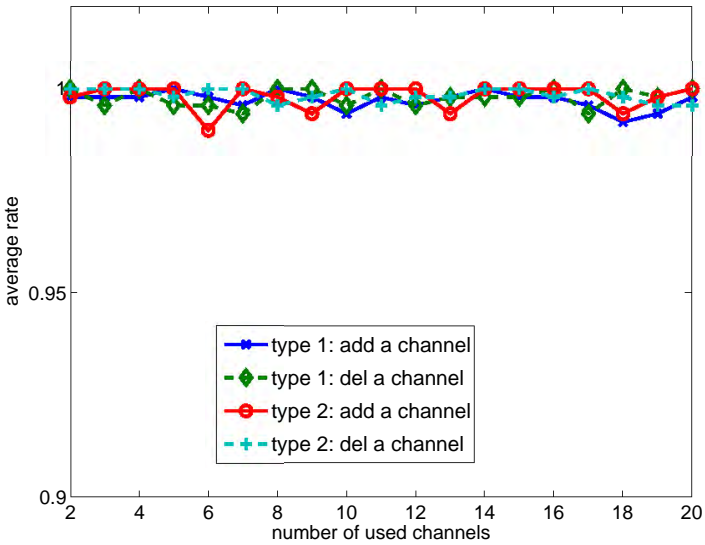

(a) Sampling $15 \%$ elements of $\Delta B$

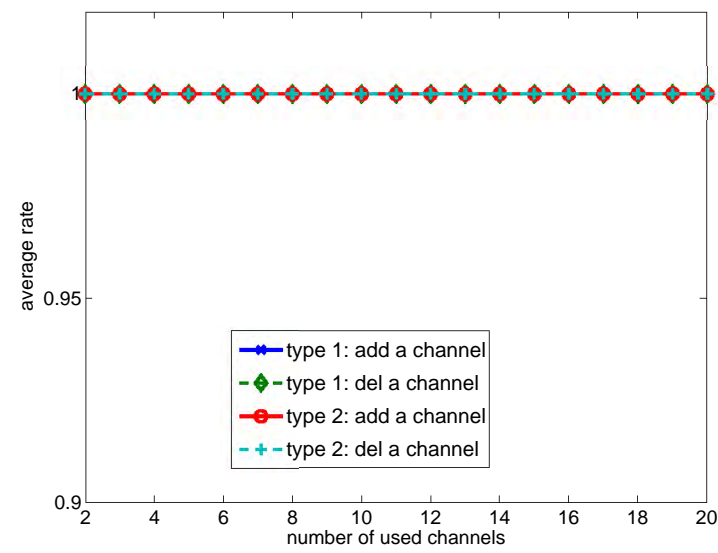

(b) Sampling $30 \%$ elements of $\Delta B$

Fig. 2. Average rate (500 runs each point) of correct recovery for $\mathrm{p}=30, \mathrm{n}=100$, $\mathrm{m}=20$ and $\mathrm{SNR}=30$.

and occupancies evolve over time. In the dynamic setting, our method recovers each change quickly for a very small number of measurements. In the simulation, we can see that the proposed method is effective in different SNRs, the number of channels, and the number of received CR reports .

\section{ACKNOWLEDGEMENTS.}

The work of Z. Wen was supported in part by NSF DMS0439872 through UCLA IPAM. The work of S. Li was supported in part by Rice Brown Undergraduate Research Award. The work of W. Yin was supported in part by NSF CAREER Award DMS-07-48839, ONR Grant N00014-08-11101, ARO/ARL grant W911NF-09-1-0383, and an Alfred P. Sloan Research Fellowship. The work of J.Meng and Z. Han was partially supported in part by NSF CNS-0910461, CNS0905556, CNS-0953377, and ECCS-1028782.

\section{REFERENCES}

[1] S. Haykin, "Cognitive Radio: Brain-Empowered Wireless Communications," IEEE Journal on Selected areas in Communications, Volume 23, Issue 2, Pages: 201-220, February 2005.

[2] E. Hossain, D. Niyato, and Z. Han, Dynamic Spectrum Access in Cognitive Radio Networks, Cambridge University Press, UK, 2009.

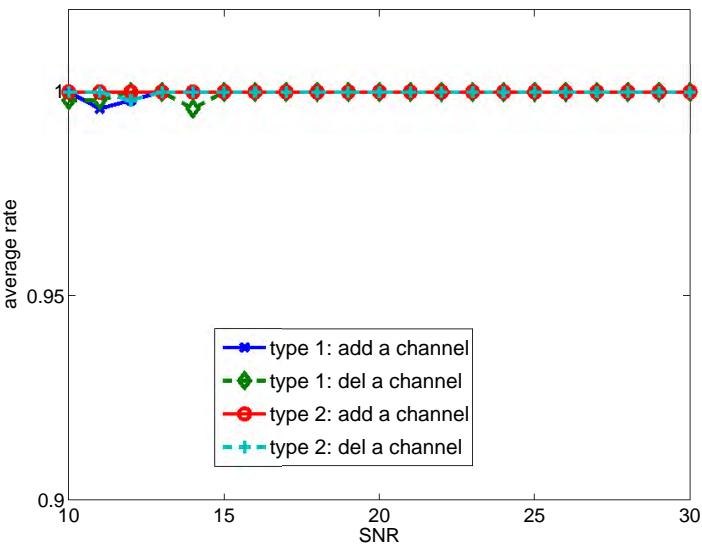

(a) Four occupied channels

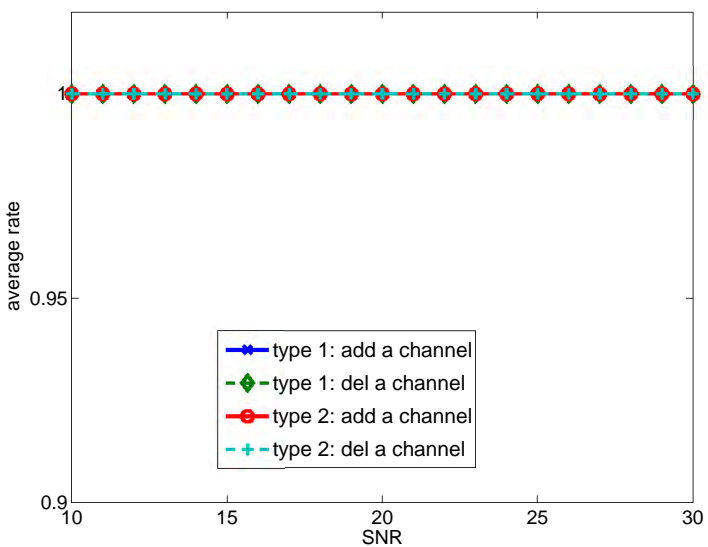

(b) Eight occupied channels

Fig. 3. Average rate (500 runs each point) of correct recovery for $\mathrm{p}=30, \mathrm{n}=100$, $\mathrm{m}=20$. Sampling $30 \%$ elements of $\Delta B$

[3] E. J. Candès and B. Recht, "Exact Low-Rank Matrix Completion via Convex Optimization," in Proc. Communication, Control, and Computing, 46th Annual Allerton Conference, Monticello, IL, September 2008.

[4] J. Meng, W. Yin, H. Li, E. Hossain, and Z. Han, "Collaborative Spectrum Sensing from Sparse Observations Using Matrix Completion for Cognitive Radio Networks," submitted to IEEE ICASSP'10 September 2009.

[5] Alfred M. Bruckstein, David L. Donoho, and Michael Elad, "From Sparse Solutions of Systems of Equations to Sparse Modeling of Signals and Images," SIAM Review, Volume 51, Issue 1, Pages: 34-81, February 2009.

[6] E. J. Candès and B. Recht, "Exact low-rank matrix completion via convex optimization," in Proc. Communication, Control, and Computing, 46th Annual Allerton Conference, Monticello, IL, September 2008.

[7] M. Yuan, Y. Lin. "Model selection and estimation in regression with grouped variables", Journal of the Royal Statistical Society: Series B, 68(1), 4967, 2006.

[8] E. van den Berg, M. Friedlander. "Joint-sparse recovery from multiple measurements", arXiv:0904.2051v1, 2009.

[9] S. Ma, D. Goldfarb, and L. Chen, "Fixed point and Bregman iterative method for matrix rank minimization," Technical Report, Department of IEOR, Columbia University, 2008

[10] D. Coppersmith, S. Winograd, "Matrix multiplication via arithmetic progressions", Journal of Symbolic Computation, 9, 251-280, 1990.

[11] A. Ghasemi and E. S. Sousa, "Collaborative spectrum sensing for opportunistic access in fading environments," in Proc. of IEEE Symp. New Frontiers in Dynamic Spectrum Access Networks, Baltimore, USA, Nov. 2005, pp. 131136.

[12] E. Visotsky, S. Kuffner, and R. Peterson, "On collaborative detection of TV transmissions in support of dynamic spectrum sensing," in Proc. 
of IEEE Symp. New Frontiers in Dynamic Spectrum Access Networks, Baltimore, USA, Nov. 2005, pp. 338356.

[13] B. Wang, K. J. R. Liu, and T. Clancy, "Evolutionary game framework for behavior dynamics in cooperative spectrum sensing," in Proc. of IEEE Global Communication Conference, New Orleans, USA, Dec. 2008.

[14] W. Zhang and K. B. Letaief, "Cooperative spectrum sensing with transmit and relay diversity in cognitive networks," IEEE Trans. Wireless Commun., vol. 7, no. 12, p.p. 4761-4766, December 2008.

[15] S. M. Mishra, A. Sahai, and R. Brodersen, "Cooperative sensing among cognitive radios," in Proc. of Int. Conf. on Communications, Istanbul, Turkey, Jun. 2006, pp. 16581663.

[16] C. Sun, W. Zhang, and K. B. Letaief, "Cooperative spectrum sensing for cognitive radios under bandwidth constraint," in Proc. of IEEE Wireless Communications and Networking Conf., Hong Kong, Mar. 2007.

[17] L. S. Cardoso, M. Debbah, P. Bianchi, and J. Najim, "Cooperative spectrum sensing using random matrix theory," in Proc. of International Symposium on Wireless Pervasive Computing, Santorini, Greece, May 2008.

[18] Y. Wang, Z. Tian, and C. Feng, "A Two-Step Compressed Spectrum Sensing Scheme for Wideband Cognitive Radios", in Proc. of IEEE Global Telecommunications Conference, 2010.

[19] S. Hong, "Multi-Resolution Bayesian Compressive Sensing for Cognitive Radio Primary User Detection", in Proc. of IEEE Global Telecommunications Conference, 2010.

[20] F. Zeng, C. Li, and Z. Tian, "Distributed Compressive Spectrum Sensing in Cooperative Multihop Cognitive Networks," IEEE Journal of Selected Topics in Signal Processing, to appear.

[21] Z. Tian; E. Blasch, W. Li, G. Chen, and X. Li, "Performance evaluation of distributed compressed wideband sensing for cognitive radio networks," in Proc. of 11th International Conference on Information Fusion, 2008

[22] M. S. Asif and J. Romberg, "Dynamic Updating for $l_{1}$ Minimization," IEEE Journal of Selected Topics in Signal Processing, Vol. 4 , No. 2, p.p. 421-434, April, 2010.

[23] M. S. Asif and J. Romberg, "Dynamic updating for sparse time varying signals," 43rd Annual Conference on Information Sciences and Systems (CISS), 2009.

[24] M. S. Asif and J. Romberg, "Streaming measurements in compressive sensing: 11 filtering," in Proc. of 42nd Asilomar Conference on Signals, Systems and Computers, 2008.

[25] N. Vaswani, "Kalman filtered Compressed Sensing," 15th IEEE International Conference on Image Processing (ICIP), 2008. 